\title{
Measuring the delayed neutrons multiplicity and kinetic parameters for the thermal induced fission of ${ }^{235} \mathrm{U},{ }^{239} \mathrm{Pu}$ and ${ }^{233} \mathrm{U}$
}

\author{
Benoit Geslot ${ }^{1}$, Alix Sardet ${ }^{2}$, Pierre Casoli ${ }^{1}$, Pierre Leconte ${ }^{3}$, Grégoire De Izarra ${ }^{1}$, Abdelhazize \\ Chebboubi $^{3}$, Grégoire Kessedjian ${ }^{3}$, Olivier Méplan ${ }^{5}$, Diane Doré ${ }^{4}$, Torsten Soldner ${ }^{6}$ and Paolo Mutti $^{6}$ \\ ${ }^{1}$ CEA, DES, IRESNE, DER, SPESI, Cadarache, F-13108 Saint Paul Lez Durance, France \\ ${ }^{2}$ CEA, DES, IRESNE, DTN, SMTA, Cadarache, F-13108 Saint Paul Lez Durance, France \\ ${ }^{3}$ CEA, DES, IRESNE, DER, SPRC, Cadarache, F-13108 Saint Paul Lez Durance, France \\ ${ }^{4}$ CEA DRF, IRFU, DPhN/LEARN, Saclay, France \\ ${ }^{5}$ CNRS IN2P3 LPSC, Grenoble, France \\ ${ }^{6}$ Institut Laue-Langevin, Grenoble, France \\ benoit.geslot@cea.fr
}

\begin{abstract}
In the normal operation of nuclear reactors, the kinetic behavior of the neutron population in the core is driven by the so-called delayed neutrons (DN). The DN yield per fission, their average lifetime and their groups' abundances are the main physical parameters used to predict the kinetic behavior of the reactor and its reactivity. The overall uncertainty associated to reactivity predictions, which is linked directly to the nuclear reactor safety margins, is thus closely dependent on a few parameters associated with DN.

Depending on the nuclear data library, DN kinetic parameters present significant discrepancies, even for major fissile isotopes such as ${ }^{235} \mathrm{U}$ or ${ }^{239} \mathrm{Pu}$. In this context, CEA has long been working for improving DN nuclear data. In 2018, CEA launched the ALDEN project (Average Lifetime of DElayed Neutrons) in the framework of a collaboration between CEA/DES, CEA/DRF, CNRS/IN2P3 (LPSC, CENBG, LPC), ENSICAEN and Caen University. This program aims at measuring the kinetics of the delayed neutrons to derive the $\mathrm{DN}$ yield, their average lifetime and abundances.
\end{abstract}

Two experimental campaigns focusing on the thermal fission of ${ }^{235} U$ were conducted in 2018 and 2019. They demonstrated the concept feasibility and gave high quality estimations of the DN multiplicity $(1.631 \pm 0.014 \%)$ and average lifetime $(8.82 \pm 0.6 \mathrm{~s})$ for ${ }^{235} \mathrm{U}$. More recently in March 2021, a more ambitious irradiation campaign was conducted on ${ }^{239} \mathrm{Pu}$ and ${ }^{233} \mathrm{U}$. This paper details the new experimental setup, which was upgraded to meet ILL safety requirements for handling plutonium. The data analysis process is presented, with a focus on the problem of dead time correction. Finally, some preliminary delayed neutron decay curves are showed and discussed.

Keywords-Delayed neutrons, fission chambers, ${ }^{3} \mathrm{He}$ proportional counters, fissile isotopes, uranium 235 , uranium 233 , plutonium 239

\section{INTRODUCTION}

$\mathrm{I}^{\mathrm{N}}$ $\mathrm{N}$ nuclear reactor physics, some fission products are referred to as "delayed neutron precursors" because they undergo a $(\beta+n)$ radioactive decay and thus produce neutrons long after the fission (from milliseconds to several minutes). In a thermal fission nuclear reactor, the DN population is roughly $0.7 \%$ of the total number of neutrons, but it still drives the kinetic behavior of the nuclear reactor. In normal operations, the prediction of the reactor's reactivity is strongly dependent on the kinetic parameters of the DN precursors. In nuclear data libraries, such as ENDF-B/VII or JEFF3.1.1, inconsistencies on some major parameters describing DN precursors can still be found [1]. This is especially true for the average lifetime of DN precursors, as well as for the average number of DN per fission (also called DN multiplicity or DN yield).

In this context, CEA has long been working on improving nuclear data evaluation of DN precursors. Among other studies, it is paramount to produce new and well documented data sets of DN kinetic parameters. This is the reason why a new experiment was designed with the aim of studying the thermal fission of several actinides of interest, among them: ${ }^{235} \mathrm{U},{ }^{239} \mathrm{Pu}$, ${ }^{233} \mathrm{U}$ and ${ }^{241} \mathrm{Pu}$.

The ALDEN project was created for that purpose within a collaboration regrouping laboratories involved in nuclear data (CEA DES and DRF, CNRS/LPSC, CNRS/CENBG, CNRS/LPC Caen, ENSICAEN and University of Caen). The principle of the experiment is to induce fissions in a miniature fission chambers loaded with a small amount of actinide (in the range of $100 \mu \mathrm{g}$ to $200 \mu \mathrm{g}$ ) and then detect DN emitted right after stopping the irradiation. An efficient fast neutrons detection system is used to monitor the decay of the DN precursors in the target. A fast beam shutter was also designed to quickly cut the beam, which allows measuring the short lived delayed neutrons.

Designed to take advantage of the PF1b cold neutron beam at ILL (Institut Laue Langevin, Grenoble, France), the experimental setup was first tested in September 2018 [1-3]. A second experimental campaign dedicated to ${ }^{235} \mathrm{U}$ was conducted in June 2019. The major outputs of these campaigns are the estimations of the DN multiplicity $(1.631 \pm 0.014 \%)$ and average lifetime $(8.82 \pm 0.6 \mathrm{~s})$ for ${ }^{235} \mathrm{U}$ thermal fission. These results are in very good agreement with the literature and have reduced associated uncertainties.

The present paper focuses on the last experimental campaign [4] performed in March 2021 on the PF1b facility [5]. Due to safety requirements for ${ }^{239} \mathrm{Pu}$, the setup was modified to add a vacuum chamber around the target. Because the DN multiplicity of ${ }^{239} \mathrm{Pu}$ is 2.5 times lower than the one of ${ }^{235} \mathrm{U}$, a key point for ensuring good quality results was to improve the signal to background ratio. This was done by reducing the 
detection background as much as possible.

After presenting the experimental setup, the last measurement campaign is detailed. The data analysis procedure is presented with a focus on the problem of dead time correction. Then, preliminary results on ${ }^{239} \mathrm{Pu}$ are shown and discussed.

\section{EXPERIMENTAL SETUP}

\section{A. Fissile targets}

The fissile targets used in this work were parallel plate miniature fission chambers (MFC) manufactured by CEA at Cadarache [8]. The fissile material (with a mass of pure isotopic actinide between 100 and $200 \mu \mathrm{g}$ of pure isotopic actinide depending on the target) was electroplated on the inner electrode of the detector (Fig. 1). The chamber's outer diameter is $12 \mathrm{~mm}$. On the miniature connector side ( $4 \mathrm{~mm}$ in diameter), a feedthrough ensures the airtightness of the detector (which encapsulate pure argon at a pressure of 12 bars).

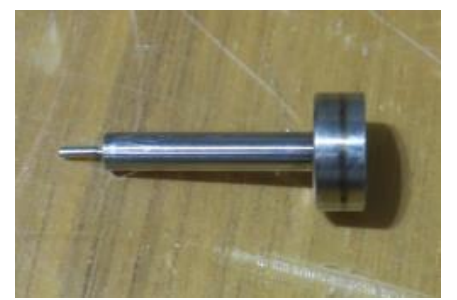

Fig. 1. View of a fissile target: a miniature fission chamber with a flat fissile deposit ( $8 \mathrm{~mm}$ in diameter) coated on its inner electrode.

\section{B. Detection system}

The LOENIEv2 detection system (named after the "long counter" LOENIE designed for the LOHENGRIN fission fragment separator at ILL) is made of high density polyethylene (PEHD) in which sixteen ${ }^{3} \mathrm{He}$ proportional counters (PC) are embedded. Neutrons are thermalized in the PEHD and then induce ${ }^{3} \mathrm{He}(\mathrm{n}, \mathrm{t})$ reactions in the PCs. The detectors are arranged in two rings: the outer ring detectors are more efficient with fast neutrons, whereas the inner ring detectors are more efficient with lower energy neutrons. This way, the total efficiency of the detection system, summed up over the 16 counters, is nearly flat in the range 0.1 to $1 \mathrm{MeV}$ of initial neutron energy (around $2 \%$ variation at maximum). This way, the total count rate induced by DN is very little dependent on their energy spectrum). A central cavity accommodates the fissile target, placed inside an airtight tubing device. Boron rubber shields cover the outer and inner faces of the PEHD blocks to cut the background thermal neutrons from entering the detection system.

First, LOENIEv2 was installed in the irradiation area, in front of the neutron guide and aligned with the beam using a laser pointer (Fig. 2a). Then, the MFC coaxial cable was placed in the central tube, with the beam stop attached to it. Its role is to absorb neutrons from the beam at the output of the detection system before they undergo scattering reactions (Fig. 2b).

Then, the ${ }^{3} \mathrm{He}$ PCs were put in place and connected to the preamplifiers by means of coaxial cables. Last, the vacuum chamber, in which the fissile target had been installed inside a glove box, was inserted in LOENIEv2 from the rear side (Fig. $2 \mathrm{c}$ and $2 \mathrm{~d})$.

\section{Beam shutters}

The beam shutters allow to stop the irradiation of the target by the cold neutrons. Their neutron capture efficiency is crucial as it drives the signal to background ratio of the measurement.

CEA developed in 2018 a fast, rotating shutter that cuts the beam in less than $10 \mathrm{~ms}$. It is made of 2 screens of boron carbide $\left(\mathrm{B}_{4} \mathrm{C}\right)$ and cadmium.

Because of concerns about a small part of alpha particles that could be converted into fast neutrons by ${ }^{12} \mathrm{C}(\alpha, n)$ reactions, it was decided to use a second beam shutter to cut these fast neutrons. Therefore, ILL developed a transversally motioned borated polyethylene block $(10 \mathrm{~cm})$, which was installed in line with the first (Fig. 3). Indeed, the background was reduced, but not as much as what was expected (see section III.D for more details).

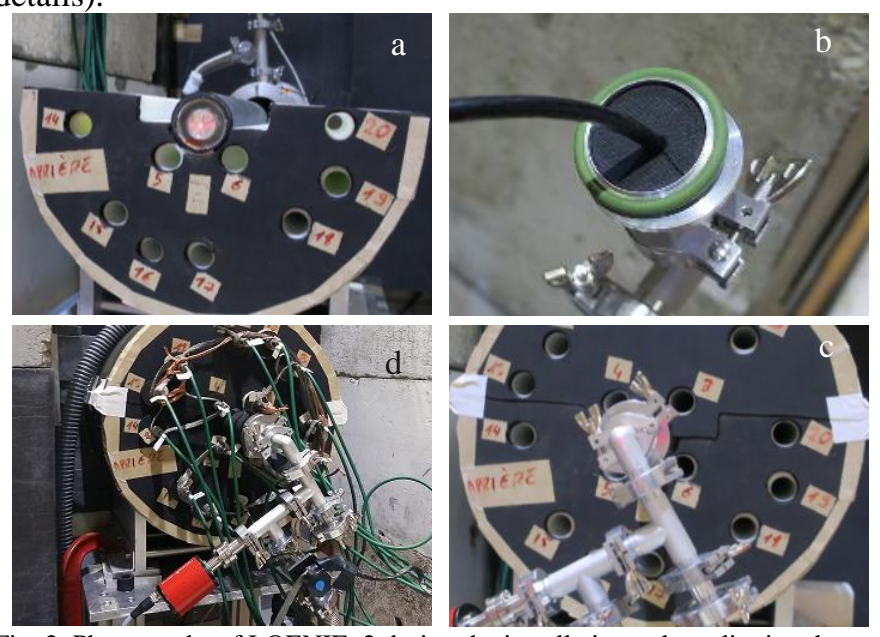

Fig. 2. Photographs of LOENIEv2 during the installation: when aligning the system with the neutron beam (a); placing the beam stop at the end of the airtight tube (b), installation of the target chamber inside the detection setup (c); after connecting all the detectors (d).

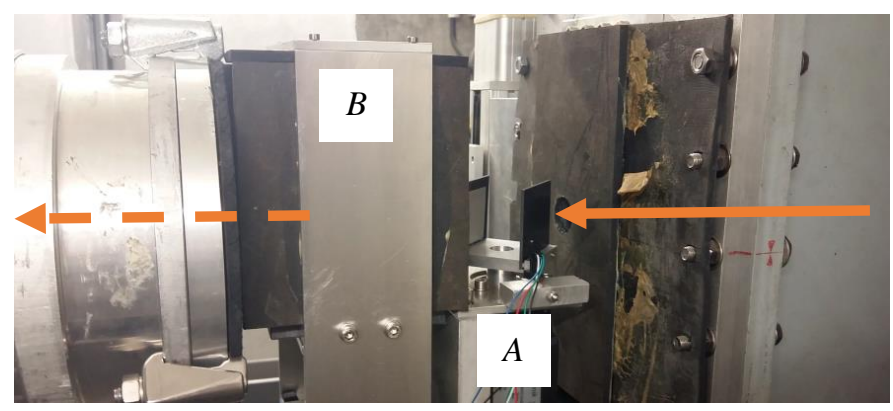

Fig. 3. Fast shutter (A) and polyethylene shutter (B) installed downstream the neutron guide line. The impinging neutron beam is featured by a solid arrow before and by a discontinuous arrow after being shaped by the shutters.

\section{Collimation and shielding}

LOENIEv2 was designed to efficiently detect neutrons in the $0.1 \mathrm{MeV}-1 \mathrm{MeV}$ energy range (its global efficiency is $22 \%$ ). If it was not for the boron rubber shielding, it would also be very efficient to detect scattered neutrons in the epithermal and thermal energy ranges. Even a small gap in the shield can lead to spurious detections, and this would dramatically degrade the signal to noise ratio (SNR).

On the front side, a wall of boron polyethylene bricks was installed as extra shielding and a central collimator shaped the beam to a diameter of $12 \mathrm{~mm}$. On the rear side, we had 
difficulties to efficiently shield the system, because of the signal cables. Nonetheless, boron rubber flanges were placed around the detectors' connectors and a tube of boron rubber covered the external part of the central device (Fig. 4).

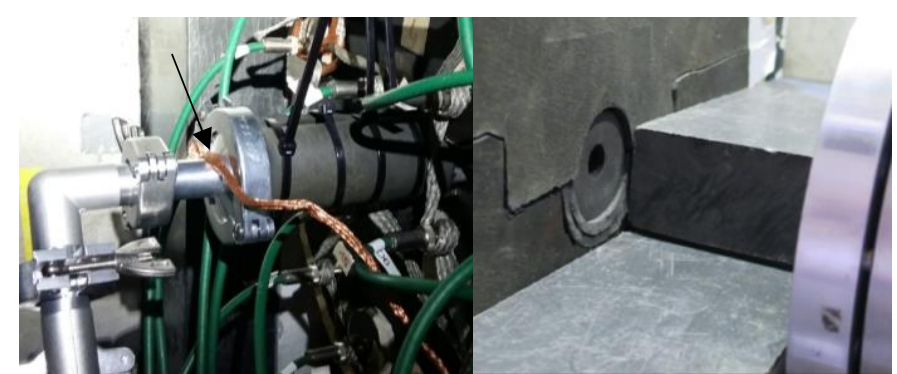

Fig. 4. Right: view of the front side of LOENIEv2 with a collimator aperture of $12 \mathrm{~mm}$ in diameter. One can see a part of the shielding wall made of borated polyethylene bricks. Left: view of the rear side of LOENIEv2 with a cylindrical shielding ( $5 \mathrm{~mm}$ of borated rubber) added for the ${ }^{233} \mathrm{U}$ campaign around the central tubing. The beam stop is placed in the iso-KF flange (see the arrow).

\section{E. Data Acquisition System}

The ILL instrument control group put together the acquisition system in 2018 for the purpose of the first experimental campaign.

A 16 channels charge sensitive preamplifier card (developed by ILL) shapes the signals of the $16{ }^{3} \mathrm{He}$ PCs. They are then fed to two synchronized digitizers (CAEN V1724, 8 channels, 14 bits $100 \mathrm{MS} / \mathrm{s}$ Flash ADC) housed in a VME crate. The digitizers run a real time digital pulse processing embedded program (CAEN DPP-PHA) to process the data flow, detect events and output their arrival time and energy (i.e. the pulses' amplitudes). Raw data buffers are stored into binary files in "list mode" for further data processing. The crate houses a third CAEN acquisition card (V1751) to record monitoring signals from the beam shutters. An optical fiber links a monitoring computer to the VME crate. This allows handling a large data rate without any loss of data buffers.

Given the large counting rate delivered by the MFC under irradiation (around $210^{5} \mathrm{~s}^{-1}$ ), it was too costly to process its signal like it was done for the ${ }^{3} \mathrm{He} \mathrm{PCs}$, and a signal reduction had to be implemented first. This is the reason why an analog amplifying stage based on a Canberra ADS7820 as broadband amplifier-discriminator was used. It shapes the current pulses and issues TTL pulses $(5 \mathrm{~V}, 100 \mathrm{~ns})$ thanks to its integrated discriminator stage.

\section{THE EXPERIMENTAL CAMPAIGN OF MARCH 2021}

\section{A. Overview of the experiments}

Acquiring high quality data on ${ }^{239} \mathrm{Pu}$ was a major objective of the last experimental campaign, conducted in March 2021. As reported in JEFF3.1, the DN yield in the ${ }^{239} \mathrm{Pu}$ thermal fission is about $0.0065(3)$, which is much smaller than the yield for ${ }^{235} \mathrm{U} 0.0162(5)$. So, the signal to noise ratio was expected to be 2.5 times lower with ${ }^{239} \mathrm{Pu}$ than with ${ }^{235} \mathrm{U}$, should the detection background be the same. Since the background has to be subtracted to the DN decay curve, it degrades in particular the estimation of the first DN group, which has the longest decay time.
Our target uncertainty associated to the estimation of ${ }^{239} \mathrm{Pu}$ $1^{\text {st }} \mathrm{DN}$ group was about 2 to $3 \%$. This implied to optimize the estimation of the background's level. First, we had to reduce its level as much as possible, by using a second beam shutter for instance. Second, we optimized the irradiation sequence for allowing a larger measurement window for estimating and monitoring the background level.

The campaign spread over March 2021 and was focused on irradiating three fissile targets $\left({ }^{235} \mathrm{U},{ }^{239} \mathrm{Pu}\right.$ and $\left.{ }^{233} \mathrm{U}\right)$ and a dummy one (see Table I).

The use of a dummy target was required for measuring the extra counting rate due to scattered neutrons (coming directly from the beam and scattered before undergoing fission, or coming from outside LOENIEv2). This was the only way to account for a bias that amounts to a few percent of the total counting rate during irradiation.

Most of beam time was used to acquire DN signals in various irradiation scenarios of interest (see table 1). The model used to describe DN precursors decay depend on whether the irradiation is large compared to the average lifetime. If so, the $\mathrm{DN}$ emission is saturated and close to its maximum level. We studied four main scenarios:

a. Short cycles $(3 \mathrm{~s} / 0.25 \mathrm{~s})$

b. Long irradiation (typically $100 \mathrm{~s} / 500 \mathrm{~s}$ )

c. Short irradiation $(5 \mathrm{~s} / 395 \mathrm{~s})$

d. Short irradiation with even irradiation and cool down (typically 10s/10s)

In the case of $235 \mathrm{U}$, only scenarios a. and b. were performed, for the sake of comparison with previous experiments and to seek for a better estimation of its $1^{\text {st }} \mathrm{DN}$ group's abundance.

TABLE I

SHORT LIST OF EXPERIMENTS CONDUCTED WITH ${ }^{235} \mathrm{U},{ }^{239} \mathrm{PU},{ }^{233} \mathrm{U}$ AND DUMMY TARGETS.

\begin{tabular}{|c|c|c|c|}
\hline Day & Target & TYPE & Type : Objective \\
\hline 5 & ${ }^{235} \mathrm{U}$ & Short $(3 \mathrm{~s} / 0.25 \mathrm{~s})$ & DN multiplicity \\
\hline 7 & ${ }^{235} \mathrm{U}$ & Long (50s/450s) & Groups abundances \\
\hline 8 & ${ }^{235} \mathrm{U}$ & Long $(100 \mathrm{~s} / 500 \mathrm{~s})$ & Groups abundances \\
\hline 8 & ${ }^{235} \mathrm{U}$ & Long irradiation & Metal foil activation \\
\hline 9 & Dummy & Long irradiation & Background \\
\hline 10 & ${ }^{239} \mathrm{Pu}$ & Short $(3 \mathrm{~s} / 0.25 \mathrm{~s})$ & DN multiplicity \\
\hline 12 & ${ }^{239} \mathrm{Pu}$ & Long (150s/750s) & Groups abundances \\
\hline 17 & ${ }^{239} \mathrm{Pu}$ & Long (50s/450s) & Groups abundances \\
\hline 19 & ${ }^{239} \mathrm{Pu}$ & Short (10s/10s) & Groups abundances \\
\hline 20 & ${ }^{239} \mathrm{Pu}$ & Short $(5 \mathrm{~s} / 5 \mathrm{~s})$ & Groups abundances \\
\hline 20 & ${ }^{239} \mathrm{Pu}$ & Short $(3 \mathrm{~s} / 3 \mathrm{~s})$ & Groups abundances \\
\hline 21 & ${ }^{239} \mathrm{Pu}$ & Long (5s/395s) & Groups abundances \\
\hline 22 & Dummy & Long irradiation & Background \\
\hline 23 & ${ }^{233} \mathrm{U}$ & Irradiation at low flux & Dead time correction \\
\hline 24 & ${ }^{233} \mathrm{U}$ & Short $(3 \mathrm{~s} / 0.25 \mathrm{~s})$ & DN multiplicity \\
\hline 25 & ${ }^{233} \mathrm{U}$ & Long $(100 \mathrm{~s} / 500 \mathrm{~s})$ & Groups abundances \\
\hline 26 & ${ }^{233} \mathrm{U}$ & Long (50s/450s) & Groups abundances \\
\hline 26 & ${ }^{233} \mathrm{U}$ & Long $(5 \mathrm{~s} / 395 \mathrm{~s})$ & Groups abundances \\
\hline 28 & ${ }^{233} \mathrm{U}$ & Short (10s/10s) & Groups abundances \\
\hline 29 & ${ }^{233} \mathrm{U}$ & Short $(5 s / 5 s)$ & Groups abundances \\
\hline 29 & ${ }^{233} \mathrm{U}$ & Short $(3 \mathrm{~s} / 3 \mathrm{~s})$ & Groups abundances \\
\hline
\end{tabular}

\section{B. Filtering parameters of the ${ }^{3}$ He proportional counters}

After the preamplifier stage, signals issued by the ${ }^{3} \mathrm{He}$ PCs were digitized, processed and filtered by the DPP-PHA (which stands for Digital Pulse Processing, Pulse Height Analysis) algorithm implemented in the CAEN V1724 digitizers. The 
overall quality of the measurements is directly linked to the parameters of the filtering algorithm (see Tab. II). The parameters used for data taking are listed in Tab II. The signal threshold, triggering pulse processing, was tested individually for each channel. This trigger threshold (TT) has a role of discriminating the pulses from the fluctuations of the baseline (statistical and/or parasitic signals).

If the TT is too low, many non-physical events are processed and recorded: that generates additional pile up and dead time. On the opposite, if the TT value is too high, then valid neutron events are not recorded. This is especially the case at high counting rate, when the energy spectrum tends to shift a bit towards the lower energies (illustration on Fig.5).

Because of the high level of electromagnetic perturbations that was observed in the PF1b environment, we had to set the TT values as high as possible, without damaging the energy spectrum of the signals. This was checked at low and high neutron fluxes. The optimum TT value was found to be 450 LSB (i.e. Least Significant Bit: unit associated to the signals after digitization).

Several channels showed noisier signals: in the energy histogram, the low energy region (under 1000 LSB) was more populated that for other channels. For those channel the TT value was increased until the energy spectrum exhibited a normal behavior.

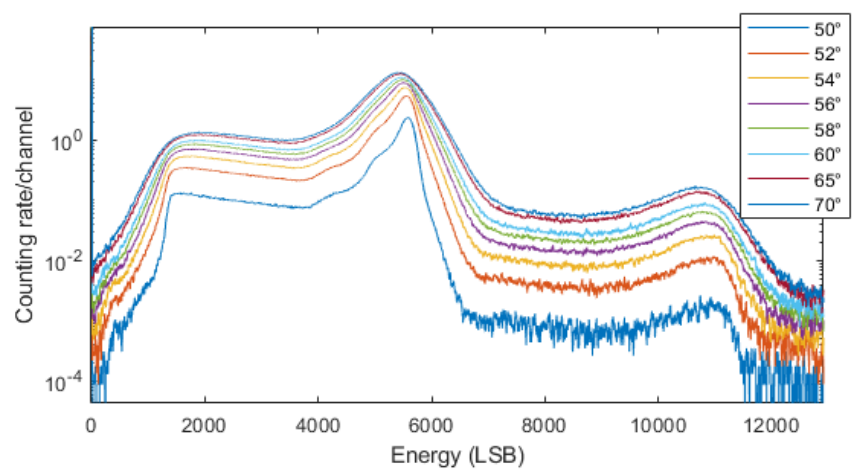

Fig. 5. Energy distributions of pulses in channel 0 (inner ring detector) for various shutter angles. Increasing the counting rate changes the shape of the energy spectrum: the peak becomes larger and slightly shifts towards low energies.

TABLE II

PSD FILTERING PARAMETERS FOR 3HE PROPORTIONAL COUNTERS

\begin{tabular}{cc}
\hline \hline Parameter & Value \\
\hline TT $($ LSB $)$ & 450 (except for \#2:550, \#5 and \#6:500) \\
Polarity & Positive \\
DC Offset & 40 \\
Smoothing & 32 \\
Trigger hold off $(\mu \mathrm{s})$ & 1.0 \\
Rise Time $(\mu \mathrm{s})$ & 0.35 \\
Decay Time $(\mu \mathrm{s})$ & 1.65 \\
Trapezoid Rise $(\mu \mathrm{s})$ & 2.0 \\
Trapezoid Flat top $(\mu \mathrm{s})$ & 1.5 \\
Baseline mean & 256 \\
Trapezoid Gain & 1 \\
Peaking delay $(\mu \mathrm{s})$ & 1.20 \\
Peak mean & 4 \\
Baseline hold off $(\mu \mathrm{s})$ & 1.0 \\
Peak Hold off $(\mu \mathrm{s})$ & 1.0 \\
\hline \hline
\end{tabular}

\section{Flux dosimetry and targets' fission rates}

Metal foil activation is a widespread technique for measuring the intensity of neutrons fluxes. In short, by measuring the activity of a particular radionuclide after the irradiation, it is possible to measure the neutron capture reaction rate $R$.

To perform metal foil activation on gold, we used an aluminum foil containing $c=0.1 \%$ of gold in mass. Because the amount of gold is low enough, any self shielding can be neglected. The foil diameter was $8 \mathrm{~mm}$, its thickness was $0.1 \mathrm{~mm}$ and its mass was $m=13.946(1) \mathrm{mg}$. It was stuck on the front face of the ${ }^{235} \mathrm{U}$ target. Then, the target was placed in the detection system the same way as usual. The metal foil was irradiated during $\mathrm{T}=15000 \mathrm{~s}$ at the maximum flux possible (the shutters wide open).

The CNRS/LPSC (Laboratory of Subatomic Physics and Cosmology, Grenoble, France) performed the gamma spectrometry measurement. The measured activity per mass unit was $A_{m}=12480(340) \mathrm{Bq} / \mathrm{g}$, which gave us a reaction rate of $3985(108) \mathrm{s}^{-1}$. Most of the uncertainty (2.7\%, at 1 sigma) is due to the efficiency calibration of the gamma spectrometry chain.

The thermal point of gold microscopic cross section is a dosimetry standard, reported at $98.7 \mathrm{~b}$ in the literature. Using this value, one can estimate an "equivalent neutron flux", i.e. the intensity of the neutron flux that would have given the same reaction rate if the neutrons were all at the thermal energy of $25 \mathrm{meV}$. The equivalent neutron flux $\Phi_{\text {eq }}$ is equal to $9.47(26)$ $10^{8} \mathrm{n} / \mathrm{cm}^{2} / \mathrm{s}$. Using this value, one can calculate a rough estimate of the fission rate $F$ in a target, by using the microscopic cross section of the fissile isotope at $25 \mathrm{meV}$ (see Table III).

TABLE III

FISSION AND CAPTURE RATES ASSOCIATED TO THE EQUIVALENT NEUTRON FLUX (AT $25 \mathrm{MEV}$ ).

\begin{tabular}{cccc}
\hline \hline Target & $\begin{array}{c}\text { Cross section } \\
\text { JEFF3.1 }(\mathrm{b})\end{array}$ & $\begin{array}{c}\text { Mass } \\
(\mu \mathrm{g})\end{array}$ & $\begin{array}{c}\text { Expected reaction rate } \\
\left(\mathrm{s}^{-1}\right)\end{array}$ \\
\hline${ }^{197} \mathrm{Au}$ & $98.7 \mathrm{~b}$ & 13.946 & 3985 \\
${ }^{235} \mathrm{U}$ & $580 \mathrm{~b}$ & $204.3^{\mathrm{a}}$ & 285600 \\
${ }^{239} \mathrm{Pu}$ & $648 \mathrm{~b}$ & $113.7^{\mathrm{a}}$ & 174500 \\
${ }^{233} \mathrm{U}$ & $504 \mathrm{~b}$ & $204^{\mathrm{b}}$ & 250100 \\
\hline \hline
\end{tabular}

aestimated based on activity measured by gamma spectrometry.

${ }^{b}$ not measured but taken equal to ${ }^{235} \mathrm{U}$ target's mass.

\section{Detection background}

In the context of this study, the detection background is defined as the total counting rate of ${ }^{3} \mathrm{He} \mathrm{PC}$ (that fall within the energy region of interest) due to particles other than prompt or delayed neutrons emitted by the target. During the measurements, the neutron beam can be either on (shutters open) or off (shutters closed). Thus, we are dealing with two types of background:

1. When the shutters were closed $\left(b_{o f f}\right)$, spurious detection of particles from the environment surrounding the detectors (cosmic rays, natural radioactivity, neutrons from surrounding experiments or coming through the beam shutter);

2. When the shutters was open $\left(b_{o n}\right)$, particles coming from interactions other than fission between the beam 
and the surrounding materials, that is mainly thermal neutrons from the beam scattered inside or outside the detection system.

The $b_{\text {off }}$ background is the one that limits the SNR associated mainly with the long lived DN (groups 1 and 2). If the irradiation cycle is long enough, $b_{\text {off }}$ can be easily estimated at the end of each cycle.

The $b_{\text {on }}$ background can introduce a bias of a few percent on the prompt neutron counting rate and thus, on the normalization of the DN activity curves.

\section{PRELIMINARY RESUlTS AND DISCUSSION}

\section{A. Data analysis}

Because each detection event in the ${ }^{3} \mathrm{He} \mathrm{PC}$ was recorded by the acquisition system, a very large amount of binary data was generated during the campaign (around 2 TB). Each irradiation cycle, called one "run" was recorded into a single binary file.

The first step was obviously to convert the binary files for better handling of the lists of events, for instance in the Matlab environment. Then, several steps were performed to convert the raw data into curves to be fitted by a mathematical model:

1. Energy scaling: the energy histogram of each channel is rescaled, so that the same energy region of interest (ROI) can be applied to all channels.

2. Background discrimination: events outside the region of interest (typically 1000 to $310^{4} \mathrm{LSB}$ ) are discarded.

3. Time synchronization: the fast shutter closing time is used as a reference time to synchronize the cycle.

4. Time histogramming: time histograms of counting rates are created. The background $b_{\text {off }}(i)$ is measured for each detector $i$ by averaging the counting rate at the end of the run (typically over the last $50 \mathrm{~s}$ ).

5. Dead time correction: histograms are dead time corrected by applying a non extendable dead time model with a parameter $\tau$ that is yet to be evaluated with precision. We know that $\tau$ is roughly equal to the veto time of the DPP-PHA algorithm: $4.7 \mu \mathrm{s}$, based on the parameters given in table 1 .

6. Summation: if no outliers are detected among the 16 histograms, they are summed up to get the overall neutron emission detection rate.

\section{B. Dead time corrections}

The so-called "dead time" phenomenon refers to the lost detections when dealing with random events, like in the case of radiations emitted by a radioactive source. In short, the measurement chain (from the detector itself to the data recording) is not always in capacity to process an incoming event. This is the case, if the waiting time between two events is shorter that a duration $\tau$, which corresponds to the standard time required for processing a detection signal.

Models can be applied to correct a posteriori the observed counting rate. Models are derived based on a few solid assumptions: events must be random and all follow the same probability distribution over time (but not necessarily Poisson) and they must be independent and not correlated.

When dealing with neutrons from fission, the last two assumptions are not perfectly true. One fission event can lead to multiple detection events, thanks to the prompt neutron multiplicity. Moreover, the time behavior of the signals is affected by correlation: this is because of the random time needed for each neutrons they reach a detector. The probability distribution of time between two events is not constant in this case. These phenomena are well known in reactor physics as they are exploited by the so-called "pile noise techniques" to determine kinetics parameters of a nuclear reactor.

The amplitude of the correlated part of the signal is proportional to the square of the detector's efficiency. This is the reason why it is usually negligible compared to the random Poisson type signal. This is not the case in LOENIEv2 because the detectors' efficiencies are large (roughly $3.5 \%$ for inner detectors and $1 \%$ for outer detectors).

This behavior was brought to light when analyzing signals at various flux levels. By putting the shutter at intermediate angles (between $50^{\circ}$ and $70^{\circ}$ ), it was possible to progressively increase the intensity of the flux on the target. The correlated signals could be observed on the waiting time histogram, i.e. the time probability between two consecutive events. If events follow a Poisson type distribution, the distribution of waiting times features an exponential curve. At low flux especially (Fig. 6, blue curve), the behavior observed is a mixed case in which the correlated signal adds up to the Poisson-type signal.

Because of this phenomenon, the observed dead time is greater than the one expected from standard dead time models.

Analysis are ongoing to implement a method to estimate the proportion of dead time due to correlated events. At high flux fortunately, it seems that the correlated signal fades out and the standard dead time correction can be applied (Fig. 6, yellow curve).

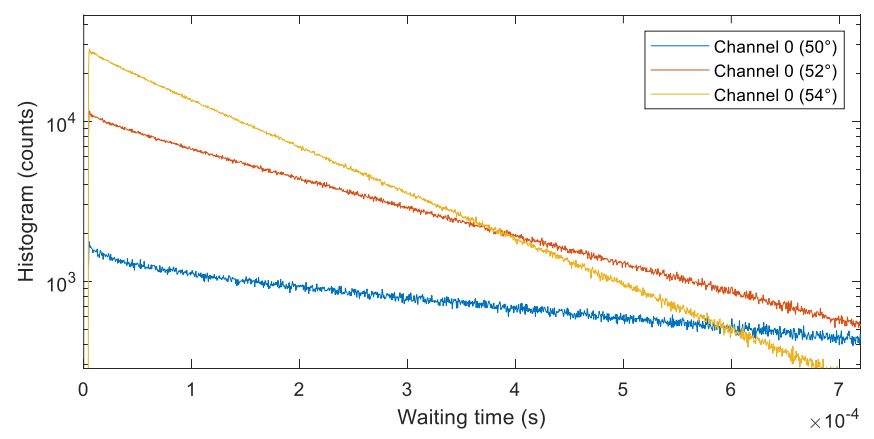

Fig. 6. Histograms of waiting times for 3 angles of the shutter (channel 0 , inner ring).

\section{Preliminary results for ${ }^{239} \mathrm{Pu}$}

The ${ }^{239} \mathrm{Pu}$ target was irradiated for 12 days in a row, from the middle of the $2^{\text {nd }}$ week until the end of the $3^{\text {rd }}$ week of the beam time. Short irradiation cycles (3s of irradiation) were first performed to obtain the counting rate just after the irradiation is stopped (Fig. 7). This is required to obtain an experimental estimate of the ratio $v_{p} / v_{d}$. This factor is then used to rescale the DN emission curves.

Most of the beam time was then used to accumulate statistics in scenarios with a long decay time, to fully deplete the target from DN precursors. Three main cases were tested with various irradiation times: $150 \mathrm{~s}$ (448 runs of $900 \mathrm{~s}$ ), $50 \mathrm{~s}$ (354 runs of $500 \mathrm{~s}$ ) and $5 \mathrm{~s}$ (398 runs of $400 \mathrm{~s}$ ). The neutron emission as 
measured by the ${ }^{3} \mathrm{He}$ PC is shown on Fig. 7 and Fig. 8. Data processing was performed as detailed in section IV.A, except for the dead time correction. The irradiation time has a clear effect on the DN activity, but also on the shape of the curve.

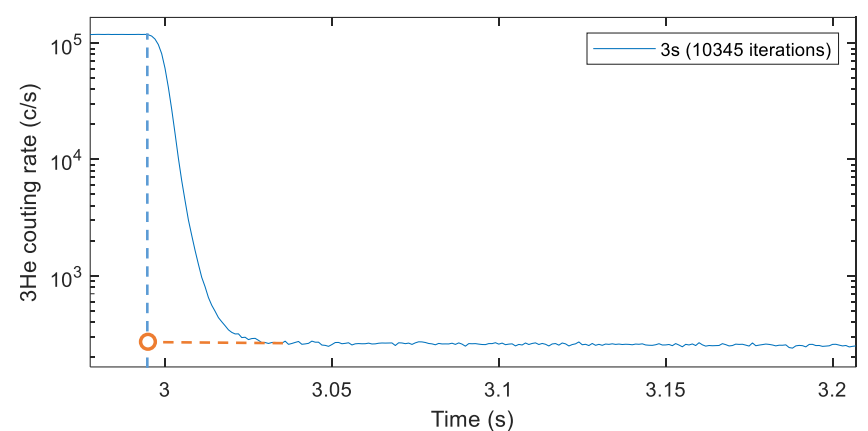

Fig. 7. Neutron emission of ${ }^{239} \mathrm{Pu}$ in the case of short irradiation cycles ( $3 \mathrm{~s}$ of irradiation).

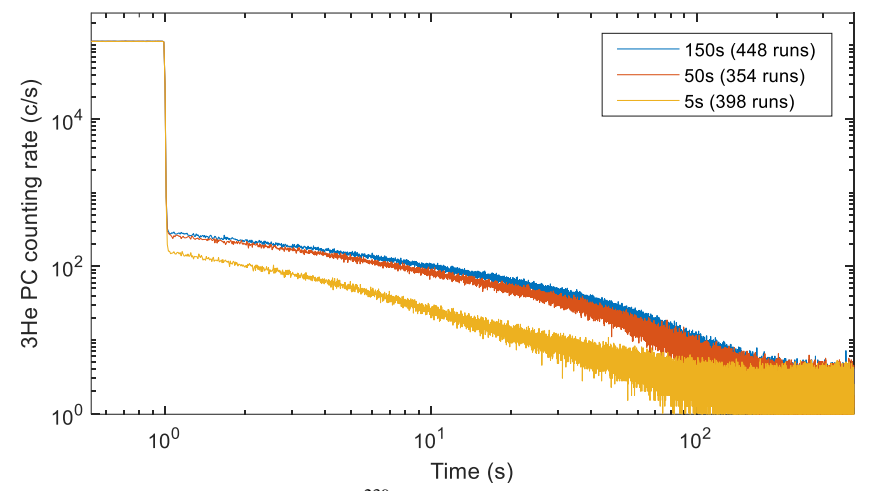

Fig. 8. Neutron emission of the ${ }^{239} \mathrm{Pu}$ target in three irradiation scenarios $(5 \mathrm{~s}$, $50 \mathrm{~s}$ and $150 \mathrm{~s}$ of irradiation). No dead time correction was taken into account.

\section{CONCLUSIONS}

CEA is actively involved in producing new measurements of DN kinetic parameters. This is the goal of the ALDEN experiments that have taken place since 2018 at ILL on the cold neutron beam PF1b. The experimental setup was designed to measure the neutron emission of a miniature fission chamber accommodated at the center of very efficient neutron detection setup. By fitting the neutron emission, it is possible to estimate the DN yield per fission, their average lifetime or the DN groups' abundances.

After two experiments dedicated to ${ }^{235} \mathrm{U}$, a more ambitious campaign performed in March 2021 focused on ${ }^{239} \mathrm{Pu}$ and ${ }^{233} \mathrm{U}$. Thirty days of beam time were allocated for this campaign and half of it was controlled remotely using the remote control tools implemented by ILL.

The campaign was a success, on nearly all technical aspects: miniature fission chambers worked perfectly, as well as the data acquisition system. The SNR was improved thanks to a careful work on the detection background (reduced down to $2 \mathrm{c} / \mathrm{s}$ ). In the future, the irradiation conditions should be reproducible, notably the alignment of the detection setup with the beam.

The comprehensive data analysis is still to be performed, and final results will be published elsewhere, but preliminary results show that the measurements' quality is very satisfactory.

\section{ACKNOWLEDGMENT}

This work was partly funded by I3P Institute gathering CEA, EdF and Framatome, and by the NEEDS project gathering CEA and CNRS. The authors would like to thank warmly the ILL staff members who were actively involved in the preparation of ALDEN experiments.

\section{REFERENCES}

[1] D. Foligno, "New evaluation of delayed-neutron data and associated covariances", $\mathrm{PhD}$ thesis, N²017AIXM0001/001ED62. Aix Marseille Université, France, Oct. 2019.

[2] P. Leconte, A. Billebaud, G. De Izarra, D. Doré, D. Foligno, B. Geslot, G. Kessedjian, U. Köster, F.R. Lecolley, J-L. Lecouey, X. Ledoux, L. Mathieu, O. Serot, N. Marie-Nourry, T. Soldner, (2019) "Absolute yield and time dependence of delayed neutron emission in $235 \mathrm{U}(\mathrm{n}, \mathrm{f})$ and $239 \mathrm{Pu}(\mathrm{n}, \mathrm{f})$ ", Institut Laue-Langevin. (ILL) doi:10.5291/ILL-DATA.3-07-380

[3] B. Geslot, D. Bernard, A. Billebaud, P. Casoli, G. De Izarra, M. Diakaki, D. Doré, D. Foligno, D. Garnier, G. Kessedjian, U. Koester, F-R. Lecolley, P. Leconte, J-L. Lecouey, X. Ledoux, L. Matthieu, O. Méplan, A. SardetO. Serot, T. Soldner (2019). "Absolute yield and kinetic parameters of delayed neutrons from the neutron-induced fission of Pu-239" Institut Laue-Langevin (ILL) doi:10.5291/ILL-DATA.3-07-385

[4] P. Leconte, D. Bernard, A. Billebaud, P. Casoli, A. Chebboubi , G. De Izarra, D. Doré, D. Garnier, B. Geslot, G. KessedjianU. Koester, F-R. Lecolley, J-L. Lecouey, X. Ledoux, N. Marie, L. Mathieu Ludovic, O. Méplabn, A. Sardet; O. Serot, T. Soldner, (2021). Absolute yield and kinetic parameters of delayed neutrons from the neutron-induced fission of 233U. Institut Laue-Langevin (ILL) doi:10.5291/ILL-DATA.3-07-396

[5] H. Abele et al., Nucl. Instr. Meth. A 562 (2006) 407.

[6] M. A. Lone et al, Nucl. Instr. Meth. 174 (1980) 521.

[7] B. Geslot, P. Leconte, G. de Izarra, D. Foligno, O. Serot, A. Chebboubi, A. Billebaud, L. Mathieu, N. Marie, O. Méplan, F.-R. Lecolley, J.-L. Lecouey, U. Koester and T. Soldner. "A new experiment at ILL for measuring the kinetic behavior of delayed neutron precursors from thermal induced fission of 235U'. IEEE. Trans. Nucl. Sci. To be published.

[8] B. Geslot, et al., "Development and manufacturing of special fission chambers for in-core measurement requirements in nuclear reactors," 2009 1st Int. Conf. on Advancements in Nuclear Instrumentation, Measurement Methods and their Applications. ANIMMA, 7-10 June 2009, Marseille, France. 